\title{
The impact of obesity on hospitalized patients with ulcerative colitis
}

\author{
Mohammed El-Dallala,b, Daniel J. Stein', Yoshihiko Raitad, Joseph D. Feuerstein ${ }^{\mathrm{b}}$
}

Cambridge Health Alliance and Harvard Medical School, Cambridge, MA; Beth Israel Deaconess Medical Center and Harvard Medical School, Boston, MA; Brigham and Women's Hospital and Harvard Medical School, Boston, MA; Massachusetts General Hospital, Harvard Medical School, Boston, MA, USA

\begin{abstract}
Background Obesity is the fifth leading risk factor for mortality in the world and it has increased among patients with ulcerative colitis in recent years. We examined the impact of obesity on the hospitalized patients admitted primarily with a diagnosis of ulcerative colitis.

Methods We used the National Inpatient Sample data for the year 2016 to identify patients with ulcerative colitis and compared obese and non-obese patients in terms of length of hospital stay, total charges, and mortality. We used multiple imputations to estimate missing values and survey analysis to estimate the outcomes, and we adjusted for confounders by implementing the inverse probability of treatment weighting using propensity score.
\end{abstract}

Results A total of 61,075 admissions with ulcerative colitis were identified. Among these, 6020 were diagnosed with obesity. Baseline hospital and patient characteristics between the 2 groups were notable for differences in age and sex. Patients with obesity were found to have a mean hospital stay longer by 0.57 days (95\% confidence interval [CI] 0.22-0.93; $\mathrm{P}=0.002)$ and charges $\$ 6341.71$ higher $(95 \%$ CI 2499.72-10,183.71; $\mathrm{P}=0.001)$ compared to non-obese patients. There was no difference in hospital mortality, with an odds ratio of 0.28 (95\%CI 0.04-2.05; $\mathrm{P}=0.212$ ).

Conclusion In a comprehensive review of inpatient admissions in 2016, primarily for ulcerative colitis, obesity was associated with a longer hospital stay and higher total charges per admission after balancing of confounders.

Keywords Ulcerative colitis, obesity, length of hospital stay, hospital cost

Ann Gastroenterol 2021; 34 (1): 1-13

\section{Introduction}

Obesity is a significant problem in the American healthcare system. It has a prevalence of $39.8 \%$ and affected approximately

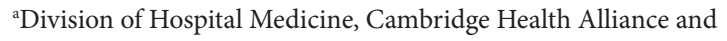
Harvard Medical School, Cambridge, MA (Mohammed El-Dallal); ${ }^{\mathrm{b} D i v i s i o n}$ of Gastroenterology, Beth Israel Deaconess Medical Center and Harvard Medical School, Boston, MA (Mohammed El-Dallal, Joseph D. Feuerstein); 'Division of Gastroenterology, Hepatology and Endoscopy, Brigham and Women's Hospital and Harvard Medical School, Boston, MA (Daniel J. Stein); ${ }^{\mathrm{d} D e p a r t m e n t ~ o f ~ E m e r g e n c y ~}$ Medicine, Massachusetts General Hospital, Harvard Medical School, Boston, MA (Yoshihiko Raita), USA

\section{Conflict of Interest: None}

Correspondence to: Mohammed El-Dallal, MD, Division of Gastroenterology, Beth Israel Deaconess Medical Center, 110 Francis St 8e Gastroenterology, Boston MA 02215, USA,

e-mail: meldalla@bidmc.harvard.edu

Received 30 June 2020; accepted 10 September 2020; published online 27 January 2021

DOI: https://doi.org/10.20524/aog.2021.0592
93.3 million US adults in 2015-2016 [1]. It has been identified as the fifth leading global risk factor for mortality in the world [2]. Despite the historically lower body mass index (BMI) in patients with ulcerative colitis (UC) compared to the general population; there is an increasing trend in obesity rates among patients with UC in recent years $[3,4]$.

Obesity has been associated with an increase in inflammatory markers such as C-reactive protein, erythrocyte sedimentation rate, and other cytokines [5-7]. There are multiple potential pathways by which obesity may worsen outcomes in UC. A reduction in bacterial diversity, with accompanying dysbiosis of the gut, and higher intestinal permeability may contribute to worsening chronic inflammatory diseases by inducing a persistent low-grade inflammatory process [6]. Some studies also suggest direct involvement of adipose tissue and mesenteric fat in creating a proinflammatory environment for developing inflammatory bowel disease (IBD) in obese patients [7-9].

Previous work has found that BMI is linked to greater mortality and length of hospital stay, with a similar trend in patients admitted for asthma, chronic obstructive pulmonary disease, cardiovascular disease, and cancer [10]. Similarly, there is an association between obesity and worse clinical outcomes in patients with autoimmune diseases, such as rheumatoid 
arthritis, psoriasis, and multiple sclerosis [11-14]. However, there are only scarce and conflicting data regarding the effect of obesity on patients with UC.

While some studies showed that obesity increases healthcare burden and utilization for patients with UC compared to non-obese patients [15-17], others showed no association between obesity and UC-related emergency department visits, hospitalizations or surgical procedures [18]. Furthermore, one study found that high BMI has a favorable effect on the prognosis of patients with UC [19]. In light of the above, we examined the impact of obesity on the length of hospital stay, healthcare expenses, mortality and other clinical outcomes among hospitalized patients with UC, using the National Inpatient Sample (NIS).

\section{Materials and methods}

\section{Data source}

We used the NIS database for the year 2016. The NIS is an all-payer, annually collected abstraction of approximately 7 million hospital discharges from 46 states in addition to the District of Columbia, representing more than $97 \%$ of the US population. It is available through the Healthcare Cost and Utilization Project (HCUP) for research purposes. Since 2012, there have been changes in the sampling strategy of the NIS data to improve its precision and decrease sample error. These strategies include sampling 20\% from all participating hospitals instead of sampling hospitals, using statewide data from the HCUP to feed the NIS database, and removing all identifiers of hospitals or states, as well as data not uniformly available in all states [20].

\section{Patient selection}

Patients who had a primary diagnosis of UC were identified using the tenth revision of the International Statistical Classification of Diseases and Related Health Problems (ICD-10) code (K51) in the first 2 discharge diagnoses. This code has been used in multiple previous studies [21,22].

We excluded all subjects less than 18 years of age, and anyone with a diagnosis of underweight (ICD-10 R636) or a BMI less than $20 \mathrm{~kg} / \mathrm{m}^{2}$ (ICD-10 Z681). We then defined patients with obesity based on ICD-10 codes of obesity or a BMI $>30 \mathrm{~kg} / \mathrm{m}^{2}$ (Supplementary Table 1) and compared them to other patients in the study population.

The weighted Charlson Comorbidity Score [23-25], which unlike other indexes does not include obesity as one of its components, was calculated for all individuals in the study population, based on the ICD-10 code's definition of the included comorbidities, and was used to adjust between obese and non-obese patients. Demography (age, race, sex), median household income for the patient's ZIP Code based on the current year, the expected primary payer for the patient's hospital stay, patient location (based on a 6-category urbanrural classification scheme for the United States counties developed by the National Center for Health Statistics), whether the patient was admitted to the hospital electively, and the type of facility that the patients were transferred from (if any) were considered as potential confounders.

\section{Missing data}

The randomness of the missing values in our data was evaluated using the missing data pattern plot to recognize if there was any systematic pattern. We used multiple imputations by chained equations [26], a method recommended by the HCUP for computing missing values in the NIS database [27]. Five imputed datasets were created; we ran the analyses repeatedly on each one and then combined the results.

\section{Outcomes}

Our primary outcomes of interest were: 1) length of hospital stay; 2) total charges of hospital admission; and 3) in-hospital mortality.

We also examined other secondary outcomes including: 1) rate of IBD-related surgeries; 2) venous thromboembolism; 3) infections, including pneumonia, urinary tract infection, and any bacterial or viral infection; and 4) cardiac events during the patients' hospitalization.

\section{Statistical analysis}

$\mathrm{R}$ version 3.5.1 ( $\mathrm{R}$ foundation for statistical computing, Vienna, Austria) was used for the analysis. Descriptive statistics were used to describe the baseline hospital and patient characteristics. We used the chi-square test to compare categorical variables and 2-group analysis of variance to compare continuous variables, while the Kruskal and Fisher tests were used for variables not fitting a normal distribution. We then estimated propensity scores by applying logistic regression models to all 5 imputed datasets to estimate the log odds of the probability of being obese based on the possible confounders listed above. The common support of propensity score distribution between the obese and non-obese groups was evaluated visually using Kernel density and box-and-whiskers plots. We then calculated the inverse probability of treatment weighting (IPTW) using propensity scores. We evaluated the covariate balance between the obese and non-obese groups to confirm adequate balance with acceptable absolute standardized effect sizes below 0.1 standard deviations [28]. All analyses were performed using survey-adjusted methods, accounting for NIS-specific hospital weighting and the IPTW. As a confirmatory measure, we re-estimated propensity scores using generalized boosting modeling (GBM) and compared the final results to the previous ones. 


\section{Results}

\section{Descriptive analysis}

We identified a total of 12,215 patients $(61,075$ weighted sample size) with a primary discharge diagnosis of UC. Among those, 1204 patients (6020 weighted sample size) fulfilled the criteria for obesity based on their BMI or ICD-10 coding of obesity. These patients tended to be older ( 54 vs. 51 years), were more likely to be female ( $62 \%$ vs. $54 \%$ ), to be admitted electively to the hospital ( $28 \%$ vs. $20 \%$ ), and to have Medicare ( $37 \%$ vs. $32 \%$ ). Overall, patients with UC had a lower Weighted Charlson Comorbidity Score; however, obese patients tended to have a higher score ( 1.17 for patients with obesity vs. 0.77 for non-obese), and more specifically, a higher rate of congestive heart failure, chronic obstructive pulmonary disease, diabetes mellitus, mild liver disease and renal disease (Supplementary Table 2). Other characteristics in both groups are summarized in Table 1.

\section{Missing data}

The total amount of missing data was $6.8 \%$. Most of the missing values referred to race $(4.3 \%)$, followed by median household income for the patient's ZIP Code (1.7\%), indicator of a transfer into the hospital $(0.5 \%)$, elective admissions $(0.3 \%)$, patient location $(0.3 \%)$, expected primary payer $(0.1 \%)$, and sex $(0.1 \%)$. The missing data pattern plot showed that data appeared to be missing at random (Supplementary Fig. 1).

\section{Propensity score and IPTW}

After propensity scores had been created using a regression model on all 5 imputed datasets, the common support was reviewed using Kernel density plots and box-and-whiskers plots and was judged by the authors as sufficient to use (Supplementary Figs. 2-5). IPTW was calculated for each individual based on their propensity score. The covariate balance was achieved by using the IPTW, based on absolute standardized effect sizes below 0.1 standard deviations in all the covariates (Supplementary Tables 3,4, Supplementary Fig. 6).

\section{Outcomes}

The length of hospital stay was greater in obese patients, with a mean difference (MD) of 0.57 days ( $95 \%$ confidence interval [CI] 0.22-0.93; $\mathrm{P}=0.002$ ) compared to non-obese patients. Similarly, the MD of total charges was $\$ 6,341.71$ higher $(95 \% \mathrm{CI}$ 2499.7-10183.71; $\mathrm{P}=0.001$ ) obese patients compared to nonobese. There was no significant difference in hospital mortality, with an odds ratio (OR) of 0.28 and a wide CI that included the null value (95\%CI 0.04-2.05; $\mathrm{P}=0.212$; Fig. 1 ).

Our results from estimating propensity scores using GBM were similar, with length of stay MD 0.56 days (95\%CI 0.14-0.97; $\mathrm{P}=0.008)$, total charges MD $\$ 5657.59$ (95\%CI 1439.66-9875.52; $\mathrm{P}=0.009)$, and no significant difference in hospital mortality with OR 0.56 (95\%CI 0.08-4.07; P=0.566). Additionally, these results were consistent in subgroup analyses that stratified patients based on the presence of a simultaneous diagnosis of Crohn's disease or other types of colitis, being

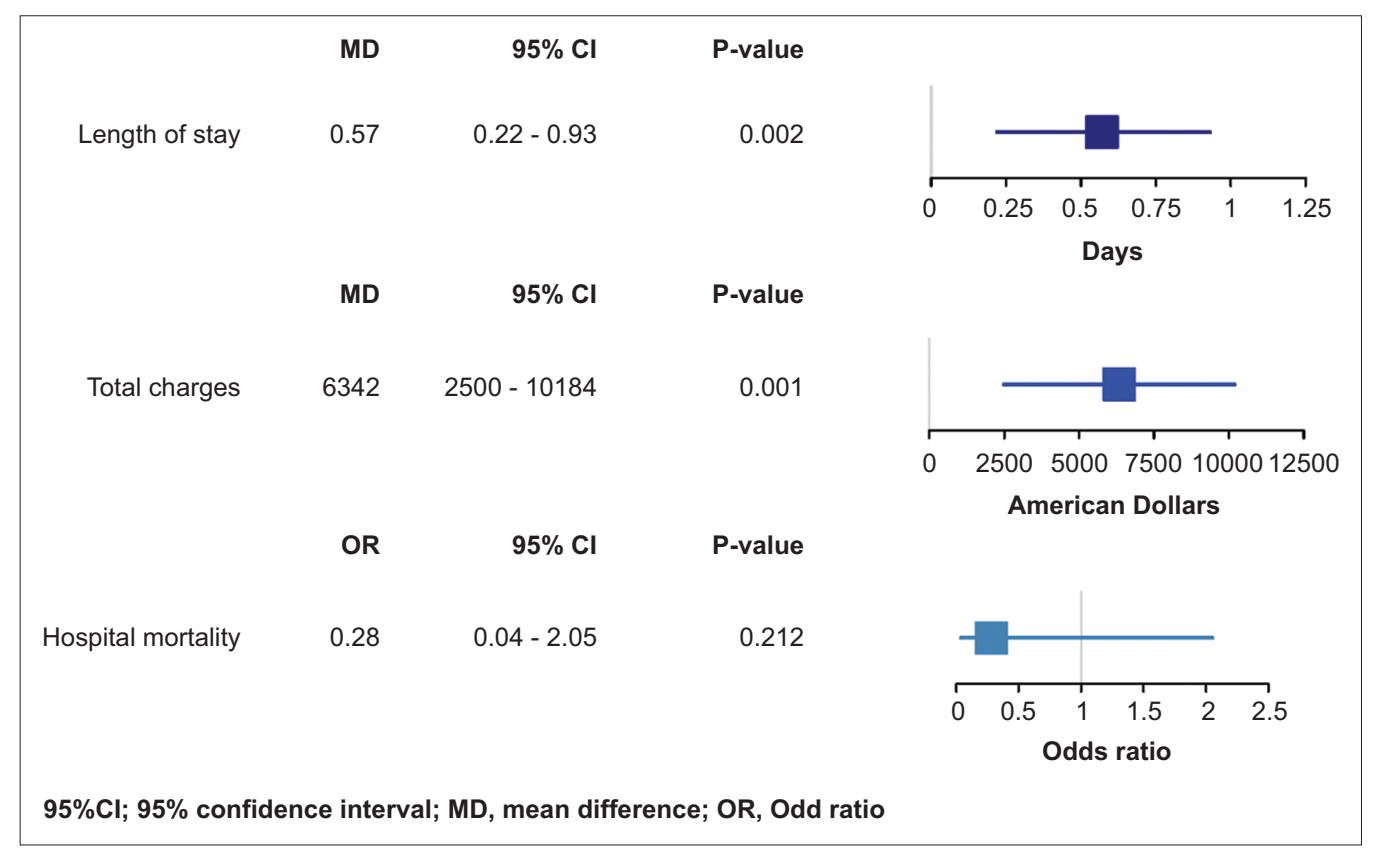

Figure 1 Outcomes plot of hospitalized patients with ulcerative colitis comparing obese and non-obese patients in relation to length of stay, total charges and hospital mortality

CI, confidence interval; $M D$, mean difference; OR, odds ratio 
Table 1 Baseline characteristics of patients with UC comparing obese and non-obese patients

\begin{tabular}{|c|c|c|c|}
\hline Characteristics & Non-obese & Obese & P-value \\
\hline Total number (weighted) & $11011(55055)$ & $1204(6020)$ & \\
\hline Age (mean (SD)) & $50.99(19.84)$ & $53.94(15.9)$ & $<0.001$ \\
\hline Elective (\%) & $10685(19.5)$ & $1665(27.7)$ & $<0.001$ \\
\hline Female (\%) & $29720(54)$ & $3720(61.9)$ & $<0.001$ \\
\hline Expected primary payer (\%) & & & 0.034 \\
\hline Medicare & $17780(32.3)$ & $2195(36.5)$ & \\
\hline Medicaid & $7825(14.2)$ & $810(13.5)$ & \\
\hline Private insurance & $25145(45.7)$ & $2625(43.6)$ & \\
\hline Self-pay & $2310(4.2)$ & $220(3.7)$ & \\
\hline No charge & $285(0.5)$ & $5(0.1)$ & \\
\hline Other & $1640(3)$ & $165(2.7)$ & \\
\hline Patient Location: NCHS Urban-Rural Code (\%) & & & 0.732 \\
\hline "Central" counties of metro areas of $\geq 1$ million population & $16305(29.7)$ & $1700(28.2)$ & \\
\hline "Fringe" counties of metro areas of $\geq 1$ million population & $15515(28.3)$ & $1655(27.5)$ & \\
\hline Counties in metro areas of $250,000-999,999$ population & $10895(19.9)$ & $1230(20.4)$ & \\
\hline Counties in metro areas of $50,000-249,999$ population & $4930(9)$ & $565(9.4)$ & \\
\hline Micropolitan counties & $4215(7.7)$ & $525(8.7)$ & \\
\hline 6Not metropolitan or micropolitan counties & $2995(5.5)$ & $345(5.7)$ & \\
\hline Race (\%) & & & 0.012 \\
\hline White & $40075(76)$ & $4375(76.7)$ & \\
\hline Black & $5110(9.7)$ & $645(11.3)$ & \\
\hline Hispanic & $4700(8.9)$ & $495(8.7)$ & \\
\hline Asian or Pacific Islander & $915(1.7)$ & $25(0.4)$ & \\
\hline Native American & $255(0.5)$ & $30(0.5)$ & \\
\hline Other & $1680(3.2)$ & $135(2.4)$ & \\
\hline Indicator of a transfer into the hospital & & & 0.484 \\
\hline Not transferred in & $51969.9(94.8)$ & $5705(95.3)$ & \\
\hline Transferred in from a different acute care hospital & $2130(3.9)$ & $225(3.8)$ & \\
\hline Transferred in from another type of health facility & $715(1.3)$ & $55(0.9)$ & \\
\hline Median household income for patient's ZIP Code (\%) & & & 0.026 \\
\hline $0-25^{\text {th }}$ percentile & $12165(22.5)$ & $1435(24.1)$ & \\
\hline $26^{\text {th }}$ to $50^{\text {th }}$ percentile & $13170(24.4)$ & $1580(26.5)$ & \\
\hline $51^{\text {st }}$ to $75^{\text {th }}$ percentile & $14215(26.3)$ & $1595(26.7)$ & \\
\hline $76^{\text {th }}$ to $100^{\text {th }}$ percentile & $14505(26.8)$ & $1355(22.7)$ & \\
\hline Weighted Charlson score (mean (SD)) & $0.77(1.37)$ & $1.17(1.59)$ & $<0.001$ \\
\hline Concurrent diagnosis of CD and other colitis (\%) & $2125(3.9)$ & $300(5.0)$ & 0.063 \\
\hline Overweight (\%) & $775(1.4)$ & $270(4.5)$ & $<0.001$ \\
\hline Older than 65 years (\%) & $16025(29.1)$ & $1775(29.5)$ & 0.791 \\
\hline
\end{tabular}

CD, Crohn's disease; NCHS, National Center for Health Statistics; SD, standard deviation

overweight, or being younger than 65 years (Supplementary Figs. 7,8).

For the secondary outcomes, there was no statistical differences between the 2 groups in the rate of IBD-related surgery, venous thromboembolic events or the rate of infections, with ORs of 1.12 (95\%CI 0.88-1.43; $\mathrm{P}=0.354), 1.21$ (95\%CI 0.94-1.55; $\mathrm{P}=0.13$ ), and 1.24 (95\%CI 0.96-1.6; $\mathrm{P}=0.1$ ), respectively. On the other hand, obese patients appeared to 
have a higher risk of having myocardial infarction, with an OR of 1.26 (95\%CI 1.03-1.55; $\mathrm{P}=0.025)$.

\section{Discussion}

In this article, we studied the impact of obesity on the outcomes of hospitalized patients with UC using the NIS database. Our results show that obesity is associated with a greater length of hospital stay and total hospital charge per admission, even after adjustment for other comorbidities. Nevertheless, we did not find a significant difference in hospital mortality between obese and non-obese patients.

This effect is likely to be multifactorial given the complex nature of obesity, which could affect both the medical and surgical management of patients with UC. The proinflammatory process induced by obesity may complicate the clinical picture of patients with UC, interfere with biological treatment dosing, or worsen some of the side effects of systematic steroid use, such as hyperglycemia. In addition, obesity has been found to increase IBD-related surgery in patients with UC [4], while multiple studies have shown that obesity is an independent risk factor for postoperative complications, including wound infections, postoperative myocardial infarction, stoma problems, and other postsurgical complications [29-31], that might contribute to an increase in the length of hospital stay and hospital charges.

These results are in alignment with other studies that focused on outpatient settings and showed an increase in annual charges, the number of hospitalized days per year, and surgical complications $[4,15,32]$. A cohort study that used the Nationwide Readmissions Database to estimate the burden of obesity in patients with IBD showed that the obese group spent a median of 8 days with a median charge of $\$ 17,277$, compared to 5 days and $\$ 11,847$ in the non-obese group [15]. Nonetheless, Christian et al found that obesity is one of the predictors of early hospital readmission within 30 days for patients with IBD and increased readmissions with an OR of 1.72 (95\%CI 1.20-2.47) [16].

Our study was based on data obtained from a discharge sample of hospitalized patients, and interpreting the results out of this context should be avoided. Furthermore, we used the diagnostic ICD-10 codes to identify the patient population, and our results are dependent on the accuracy of these codes; however, we used verified codes that have been used in many other studies. Identifying patients with obesity was a challenging part of our project, as obesity is known for under-coding [33]. Obesity defined by BMI has been accepted in population-based studies and was found to be a better predictor for cardiovascular mortality compared to other obesity indicators [34,35]. We also used ICD-10 code E66 to identify obesity, which has been verified in a study and found to have a specificity of $99 \%$ and a sensitivity of $7.8 \%$ [33].

Patients with UC already have an increased risk of coronary artery disease [36-38], diabetes [39] and stroke [40], regardless of their body weight. In our study, we showed that obesity increases the healthcare costs of hospitalized patients with UC, probably by worsening their medical and surgical condition. Further studies are needed to study the etiology of this relation and the best way to manage it using a multidisciplinary approach, including nutrition, lifestyle changes, and possibly altering some of the UC-related medications.

In conclusion, in a comprehensive review of inpatient admissions in 2016, primarily for UC, obesity was associated with a longer hospital stay and higher total charges per admission after balancing of confounders, including demographics and comorbidities. Future studies are needed to better understand obesity's impact on disease management and to identify methods for improving care in patients with UC.

\section{Acknowledgment}

This work was conducted with support from Harvard Catalyst | The Harvard Clinical and Translational Science Center (National Center for Research Resources and the National Center for Advancing Translational Sciences, National Institutes of Health Award UL1 TR002541) and financial contributions from Harvard University and its affiliated academic health care centers. The content is solely the responsibility of the authors and does not necessarily represent the official views of Harvard Catalyst, Harvard University and its affiliated academic health care centers, or the National Institutes of Health

\section{Summary Box}

\section{What is already known:}

- The proinflammatory effect of obesity can affect the clinical course of chronic and inflammatory diseases

- The prevalence of obesity has increased in patients with ulcerative colitis (UC) in recent years

- There are conflicting data regarding the effect of obesity on the clinical outcomes of patients with UC

\section{What the new findings are:}

- Hospitalized patients with UC and obesity have a higher rate of comorbidities compared to nonobese patients

- Obesity is associated with a longer hospital stay and greater cost for patients admitted primarily for UC, after adjustment for comorbidities and other independent factors

- The data are insufficient to support a significant different in hospital mortality between obese and non-obese patients with UC 


\section{References}

1. Hales CM, Carroll MD, Fryar CD, Ogden CL. Prevalence of obesity among adults and youth: United States, 2015-2016. NCHS Data Brief 2017;288:1-8.

2. World Health Organization, ed. Global Health Risks: Mortality and burden of disease attributable to selected major risks. World Health Organization; 2009.

3. Back IR, Marcon SS, Gaino NM, Vulcano DSB, Dorna MS, Sassaki LY. Body composition in patients with Crohn's disease and ulcerative colitis. Arq Gastroenterol 2017;54:109-114.

4. Steed H, Walsh S, Reynolds N. A brief report of the epidemiology of obesity in the inflammatory bowel disease population of Tayside, Scotland. Obes Facts 2009;2:370-372.

5. Choi J, Joseph L, Pilote L. Obesity and C-reactive protein in various populations: a systematic review and meta-analysis. Obes Rev 2013;14:232-244.

6. Cox AJ, West NP, Cripps AW. Obesity, inflammation, and the gut microbiota. Lancet Diabetes Endocrinol 2015;3:207-215.

7. Karagiannides I, Pothoulakis C. Substance P, obesity, and gut inflammation. Curr Opin Endocrinol Diabetes Obes 2009;16:47-52.

8. Bertin B, Desreumaux P, Dubuquoy L. Obesity, visceral fat and Crohn's disease. Curr Opin Clin Nutr Metab Care 2010;13:574-580.

9. Bilski J, Mazur-Bialy A, Wojcik D, et al. Role of obesity, mesenteric adipose tissue, and adipokines in inflammatory bowel diseases. Biomolecules 2019;9:780.

10. Akinyemiju T, Meng Q, Vin-Raviv N. Association between body mass index and in-hospital outcomes: Analysis of the nationwide inpatient database. Medicine (Baltimore) 2016;95:e4189.

11. Versini M, Jeandel PY, Rosenthal E, Shoenfeld Y. Obesity in autoimmune diseases: not a passive bystander. Autoimmun Rev 2014;13:981-1000.

12. Iannone F, Lopalco G, Rigante D, Orlando I, Cantarini L, Lapadula G. Impact of obesity on the clinical outcome of rheumatologic patients in biotherapy. Autoimmun Rev 2016;15:447-450.

13. Singh S, Dulai PS, Zarrinpar A, Ramamoorthy S, Sandborn WJ. Obesity in IBD: epidemiology, pathogenesis, disease course and treatment outcomes. Nat Rev Gastroenterol Hepatol 2017;14: 110-121.

14. Guerrero-García JJ, Carrera-Quintanar L, López-Roa RI, MárquezAguirre AL, Rojas-Mayorquín AE, Ortuño-Sahagún D. Multiple sclerosis and obesity: possible roles of adipokines. Mediators Inflamm 2016;2016:4036232.

15. Nguyen NH, Ohno-Machado L, Sandborn WJ, Singh S. Obesity is independently associated with higher annual burden and costs of hospitalization in patients with inflammatory bowel diseases. Clin Gastroenterol Hepatol 2019;17:709-718.

16. Christian KE, Jambaulikar GD, Hagan MN, et al. Predictors of early readmission in hospitalized patients with inflammatory bowel disease. Inflamm Bowel Dis 2017;23:1891-1897.

17. Pavelock N, Masood U, Minchenberg S, Heisig D. Effects of obesity on the course of inflammatory bowel disease. Proc (Bayl Univ Med Cent) 2019;32:14-17.

18. Seminerio JL, Koutroubakis IE, Ramos-Rivers C, et al. Impact of obesity on the management and clinical course of patients with inflammatory bowel disease. Inflamm Bowel Dis 2015;21: 2857-2863.

19. Stabroth-Akil D, Leifeld L, Pfützer R, Morgenstern J, Kruis W. The effect of body weight on the severity and clinical course of ulcerative colitis. Int J Colorectal Dis 2015;30:237-242.

20. HCUP-US NIS Overview. Available from: https://www.hcup-us. ahrq.gov/nisoverview.jsp [Accessed 5 January 2021].

21. Jones GR, Lyons M, Plevris N, et al. IBD prevalence in Lothian, Scotland, derived by capture-recapture methodology. Gut
2019;68:1953-1960.

22. Larsen MB, Njor S, Ingeholm P, Andersen B. Effectiveness of colorectal cancer screening in detecting earlier-stage disease-a nationwide cohort study in Denmark. Gastroenterology 2018;155:99-106.

23. Charlson ME, Pompei P, Ales KL, MacKenzie CR. A new method of classifying prognostic comorbidity in longitudinal studies: development and validation. J Chronic Dis 1987;40:373-383.

24. Quan H, Li B, Couris CM, et al. Updating and validating the Charlson comorbidity index and score for risk adjustment in hospital discharge abstracts using data from 6 countries. Am J Epidemiol 2011;173:676-682.

25. Radovanovic D, Seifert B, Urban P, et al; AMIS Plus Investigators. Validity of Charlson Comorbidity Index in patients hospitalised with acute coronary syndrome. Insights from the nationwide AMIS Plus registry 2002-2012. Heart 2014;100:288-294.

26. van Buuren S, Groothuis-Oudshoorn K. MICE: Multivariate imputation by chained equations in R. J Stat Softw 2011;45:1-67.

27. Houchens R. Missing data methods for the NIS and the SID. 2015. HCUP Methods Series Report \# 2015-01 ONLINE. January 22, 2015. U.S. Agency for Healthcare Research and Quality. Available from: http://www.hcup-us.ahrq.gov/reports/methods/methods.jsp [Accessed 5 January 2021].

28. Austin PC. An introduction to propensity score methods for reducing the effects of confounding in observational studies. Multivariate Behav Res 2011;46:399-424.

29. Gendall KA, Raniga S, Kennedy R, Frizelle FA. The impact of obesity on outcome after major colorectal surgery. Dis Colon Rectum 2007;50:2223-2237.

30. Bamgbade OA, Rutter TW, Nafiu OO, Dorje P. Postoperative complications in obese and nonobese patients. World J Surg 2007;31:556-560.

31. Glance LG, Wissler R, Mukamel DB, et al. Perioperative outcomes among patients with the modified metabolic syndrome who are undergoing noncardiac surgery. Anesthesiology 2010;113:859-872.

32. Boutros M, Maron D. Inflammatory bowel disease in the obese patient. Clin Colon Rectal Surg 2011;24:244-252.

33. Martin BJ, Chen G, Graham M, Quan H. Coding of obesity in administrative hospital discharge abstract data: accuracy and impact for future research studies. BMC Health Serv Res 2014;14:70.

34. Piché ME, Poirier P, Lemieux I, Després JP. Overview of epidemiology and contribution of obesity and body fat distribution to cardiovascular disease: an update. Prog Cardiovasc Dis ;61:103-113.

35. Ortega FB, Sui X, Lavie CJ, Blair SN. Body mass index, the most widely used but also widely criticized index: would a criterion standard measure of total body fat be a better predictor of cardiovascular disease mortality? Mayo Clin Proc 2016;91:443-455.

36. Bernstein CN, Wajda A, Blanchard JF. The incidence of arterial thromboembolic diseases in inflammatory bowel disease: a population-based study. Clin Gastroenterol Hepatol 2008;6:41-45.

37. Yarur AJ, Deshpande AR, Pechman DM, Tamariz L, Abreu MT, Sussman DA. Inflammatory bowel disease is associated with an increased incidence of cardiovascular events. Am J Gastroenterol 2011;106:741-747.

38. Gandhi S, Narula N, Marshall JK, Farkouh M. Are patients with inflammatory bowel disease at increased risk of coronary artery disease? Am J Med 2012;125:956-962.

39. Kang EA, Han K, Chun J, et al. Increased risk of diabetes in inflammatory bowel disease patients: a nationwide populationbased study in Korea. J Clin Med 2019;8:343.

40. Singh S, Singh H, Loftus EV Jr, Pardi DS. Risk of cerebrovascular accidents and ischemic heart disease in patients with inflammatory bowel disease: a systematic review and meta-analysis. Clin Gastroenterol Hepatol 2014;12:382-393.e1. 


\section{Supplementary material}

Supplementary Table 1 ICD-10 codes used in the study

\begin{tabular}{|c|c|}
\hline ICD-10 codes & Interpretation \\
\hline $\mathrm{K} 51^{*}$ & Ulcerative colitis \\
\hline Z681 & BMI of 19.9 or less \\
\hline R636 & $\begin{array}{l}\text { Diagnosis of } \\
\text { underweight }\end{array}$ \\
\hline Z68230, Z6831, Z6832, Z6833, Z6834, & $\begin{array}{l}\text { BMI } 30 \text { to } 70 \text { or } \\
\text { greater }\end{array}$ \\
\hline \multicolumn{2}{|l|}{ Z6835, Z6836, Z6837, Z6838, Z6839, Z6840, } \\
\hline Z6841, Z6842, Z6843, Z6844, Z6845 & \\
\hline E660, E661, E662, E668, E669 & Diagnosis of obesity \\
\hline
\end{tabular}

Supplementary Table 2 Comorbidities compared between the 2 groups

\begin{tabular}{|c|c|c|c|}
\hline Comorbidities & Non-obese & Obese & P-value \\
\hline Acute myocardial infarction (\%) & $1730(3.1)$ & $265(4.4)$ & 0.021 \\
\hline Congestive heart failure (\%) & $2115(3.8)$ & $435(7.2)$ & $<0.001$ \\
\hline Peripheral vascular disease (\%) & $2235(4.1)$ & $290(4.8)$ & 0.220 \\
\hline Cerebrovascular disease (\%) & $1105(2.0)$ & $135(2.2)$ & 0.583 \\
\hline Dementia (\%) & $1300(2.4)$ & $50(0.8)$ & 0.001 \\
\hline Chronic obstructive pulmonary disease (\%) & $7340(13.3)$ & $1290(21.4)$ & $<0.001$ \\
\hline Rheumatoid disease (\%) & $1735(3.2)$ & $220(3.7)$ & 0.345 \\
\hline Peptic ulcer disease (\%) & $710(1.3)$ & $80(1.3)$ & 0.908 \\
\hline Mild liver disease (\%) & $2260(4.1)$ & $365(6.1)$ & 0.002 \\
\hline Diabetes without complications (\%) & $6255(11.4)$ & $1420(23.6)$ & $<0.001$ \\
\hline Diabetes with complications (\%) & $1090(2.0)$ & $320(5.3)$ & $<0.001$ \\
\hline Hemiplegia or paraplegia (\%) & $220(0.4)$ & $30(0.5)$ & 0.612 \\
\hline Renal disease (\%) & $2790(5.1)$ & $485(8.1)$ & $<0.001$ \\
\hline Cancer (any malignancy) (\%) & $1845(3.4)$ & $200(3.3)$ & 0.956 \\
\hline Moderate or severe liver disease (\%) & $405(0.7)$ & $40(0.7)$ & 0.781 \\
\hline Metastatic solid tumor (\%) & $365(0.7)$ & $50(0.8)$ & 0.506 \\
\hline AIDS/HIV (\%) & $95(0.2)$ & $0.0(0.0)$ & 0.149 \\
\hline Weighted Charlson score (mean (SD)) & $0.77(1.37)$ & $1.17(1.59)$ & $<0.001$ \\
\hline Pregnant (\%) & $\begin{array}{c}1330.0 \\
(2.4)\end{array}$ & $110(1.8)$ & 0.176 \\
\hline
\end{tabular}


Supplementary Table 3 Balance summary of propensity scores estimated by regression model across all imputations

\begin{tabular}{|c|c|c|c|c|}
\hline Variable & Type & Min.Diff.Un & Mean.Diff.Un & Max.Diff.Un \\
\hline prop.score & Distance & -0.399 & -0.397 & -0.396 \\
\hline AGE & Contin. & -0.164 & -0.164 & -0.164 \\
\hline ELECTIVE & Binary & -0.083 & -0.082 & -0.082 \\
\hline FEMALE & Binary & -0.079 & -0.079 & -0.078 \\
\hline \multicolumn{5}{|l|}{ Expected primary payer } \\
\hline Medicare & Binary & -0.042 & -0.041 & -0.041 \\
\hline Medicaid & Binary & 0.008 & 0.008 & 0.008 \\
\hline Private insurance & Binary & 0.021 & 0.021 & 0.021 \\
\hline Self-pay & Binary & 0.005 & 0.005 & 0.006 \\
\hline No charge & Binary & 0.004 & 0.004 & 0.004 \\
\hline Others & Binary & 0.002 & 0.002 & 0.003 \\
\hline \multicolumn{5}{|l|}{ Patient location: NCHS Urban-Rural Code } \\
\hline "Central" counties of metro areas of $\geq 1$ million population & Binary & 0.015 & 0.015 & 0.016 \\
\hline "Fringe" counties of metro areas of $\geq 1$ million population & Binary & 0.008 & 0.008 & 0.008 \\
\hline Counties in metro areas of $250,000-999,999$ population & Binary & -0.006 & -0.006 & -0.005 \\
\hline Counties in metro areas of $50,000-249,999$ population & Binary & -0.004 & -0.004 & -0.004 \\
\hline Micropolitan counties & Binary & -0.011 & -0.01 & -0.01 \\
\hline Not metropolitan or micropolitan counties & Binary & -0.003 & -0.003 & -0.002 \\
\hline \multicolumn{5}{|l|}{ RACE } \\
\hline White & Binary & -0.011 & -0.011 & -0.009 \\
\hline Black & Binary & -0.015 & -0.013 & -0.011 \\
\hline Hispanic & Binary & 0.002 & 0.003 & 0.004 \\
\hline Asian or Pacific Islander & Binary & 0.012 & 0.013 & 0.013 \\
\hline Native American & Binary & -0.002 & -0.001 & 0 \\
\hline Other & Binary & 0.008 & 0.009 & 0.01 \\
\hline \multicolumn{5}{|l|}{ Indicator of a transfer into the hospital } \\
\hline Not transferred in & Binary & -0.006 & -0.005 & -0.005 \\
\hline Transferred in from a different acute care hospital & Binary & 0.001 & 0.001 & 0.002 \\
\hline Transferred in from another type of health facility & Binary & 0.004 & 0.004 & 0.004 \\
\hline \multicolumn{5}{|l|}{ Median household income for patient's ZIP Code } \\
\hline $0-25^{\text {th }}$ percentile & Binary & -0.016 & -0.015 & -0.014 \\
\hline $26^{\text {th }}$ to $50^{\text {th }}$ percentile & Binary & -0.021 & -0.02 & -0.02 \\
\hline $51^{\text {st }}$ to $75^{\text {th }}$ percentile & Binary & -0.007 & -0.005 & -0.003 \\
\hline $76^{\text {th }}$ to $100^{\text {th }}$ percentile & Binary & 0.038 & 0.041 & 0.042 \\
\hline Weighted Charlson score & Contin. & -0.266 & -0.266 & -0.266 \\
\hline
\end{tabular}


Supplementary Table 4 Balance summary of propensity scores estimated by generalized boosting modeling

\begin{tabular}{|c|c|c|c|c|c|c|c|}
\hline Variable & $\mathrm{tx} . \mathrm{mn}$ & tx.sd & ct.mn & ct.sd & std.eff. sz & stat & P-value \\
\hline AGE & 51.451 & 18.544 & 51.276 & 19.535 & 0.009 & 0.236 & 0.814 \\
\hline ELECTIVE:0 & 0.785 & 0.411 & 0.796 & 0.403 & -0.026 & 1.056 & 0.347 \\
\hline ELECTIVE: 1 & 0.213 & 0.41 & 0.201 & 0.401 & 0.029 & NA & NA \\
\hline ELECTIVE: $<\mathrm{NA}>$ & 0.001 & 0.038 & 0.003 & 0.054 & -0.039 & NA & NA \\
\hline FEMALE:0 & 0.431 & 0.495 & 0.453 & 0.498 & -0.044 & 0.966 & 0.379 \\
\hline FEMALE:1 & 0.568 & 0.495 & 0.546 & 0.498 & 0.043 & NA & NA \\
\hline FEMALE: $<\mathrm{NA}>$ & 0.002 & 0.039 & 0.001 & 0.033 & 0.011 & NA & NA \\
\hline \multicolumn{8}{|l|}{ Expected primary payer (\%) } \\
\hline Medicare & 0.329 & 0.47 & 0.327 & 0.469 & 0.006 & 0.775 & 0.55 \\
\hline Medicaid & 0.143 & 0.35 & 0.142 & 0.349 & 0.003 & NA & NA \\
\hline Private insurance & 0.465 & 0.499 & 0.455 & 0.498 & 0.021 & NA & NA \\
\hline Self-pay & 0.035 & 0.185 & 0.041 & 0.199 & -0.032 & NA & NA \\
\hline No charge & 0.002 & 0.047 & 0.005 & 0.07 & -0.058 & NA & NA \\
\hline Others & 0.025 & 0.157 & 0.03 & 0.17 & -0.029 & NA & NA \\
\hline PAY $1:<\mathrm{NA}>$ & 0 & 0 & 0.001 & 0.034 & NA & NA & NA \\
\hline \multicolumn{8}{|l|}{ Patient location: NCHS Urban-Rural Code (\%) } \\
\hline "Central" counties of metro areas of $\geq 1$ million population & 0.292 & 0.455 & 0.295 & 0.456 & -0.007 & 1.167 & 0.322 \\
\hline "Fringe" counties of metro areas of $\geq 1$ million population & 0.277 & 0.448 & 0.281 & 0.449 & -0.007 & NA & NA \\
\hline Counties in metro areas of $250,000-999,999$ population & 0.203 & 0.402 & 0.199 & 0.399 & 0.011 & NA & NA \\
\hline Counties in metro areas of $50,000-249,999$ population & 0.094 & 0.292 & 0.09 & 0.286 & 0.014 & NA & NA \\
\hline Micropolitan counties & 0.082 & 0.274 & 0.077 & 0.267 & 0.018 & NA & NA \\
\hline Not metropolitan or micropolitan counties & 0.052 & 0.222 & 0.055 & 0.228 & -0.015 & NA & NA \\
\hline PL_NCHS: $<\mathrm{NA}>$ & 0 & 0 & 0.003 & 0.058 & NA & NA & NA \\
\hline \multicolumn{8}{|l|}{ RACE (\%) } \\
\hline White & 0.744 & 0.437 & 0.728 & 0.445 & 0.036 & 1.423 & 0.205 \\
\hline Black & 0.096 & 0.295 & 0.094 & 0.292 & 0.009 & NA & NA \\
\hline Hispanic & 0.089 & 0.285 & 0.085 & 0.279 & 0.014 & NA & NA \\
\hline Asian or Pacific Islander & 0.006 & 0.077 & 0.016 & 0.124 & -0.126 & NA & NA \\
\hline Native American & 0.004 & 0.067 & 0.005 & 0.068 & -0.003 & NA & NA \\
\hline Other & 0.024 & 0.152 & 0.03 & 0.17 & -0.042 & NA & NA \\
\hline RACE: $<N A>$ & 0.037 & 0.188 & 0.043 & 0.203 & -0.032 & NA & NA \\
\hline \multicolumn{8}{|l|}{ Indicator of a transfer into the hospital (\%) } \\
\hline Not transferred in & 0.951 & 0.215 & 0.944 & 0.23 & 0.034 & 0.354 & 0.781 \\
\hline Transferred in from a different acute care hospital & 0.033 & 0.18 & 0.039 & 0.193 & -0.031 & NA & NA \\
\hline Transferred in from another type of health facility & 0.012 & 0.109 & 0.013 & 0.112 & -0.007 & NA & NA \\
\hline \multicolumn{8}{|l|}{ Median household income for patient's ZIP Code } \\
\hline $0-25^{\text {th }}$ percentile & 0.003 & 0.058 & 0.004 & 0.066 & -0.017 & NA & NA \\
\hline $26^{\text {th }}$ to $50^{\text {th }}$ percentile (median) & 0.227 & 0.419 & 0.223 & 0.416 & 0.011 & 1.59 & 0.174 \\
\hline $51^{\text {st }}$ to $75^{\text {th }}$ percentile & 0.257 & 0.437 & 0.241 & 0.428 & 0.036 & NA & NA \\
\hline $76^{\text {th }}$ to $100^{\text {th }}$ percentile & 0.258 & 0.438 & 0.258 & 0.438 & 0 & NA & NA \\
\hline Median household income for patient's ZIP Code & 0.25 & 0.433 & 0.26 & 0.439 & -0.025 & NA & NA \\
\hline ZIPINC_QRTL: $<$ NA $>$ & 0.008 & 0.091 & 0.017 & 0.131 & -0.101 & NA & NA \\
\hline Weighted Charlson score & 0.825 & 1.399 & 0.808 & 1.395 & 0.012 & 0.385 & 0.701 \\
\hline
\end{tabular}




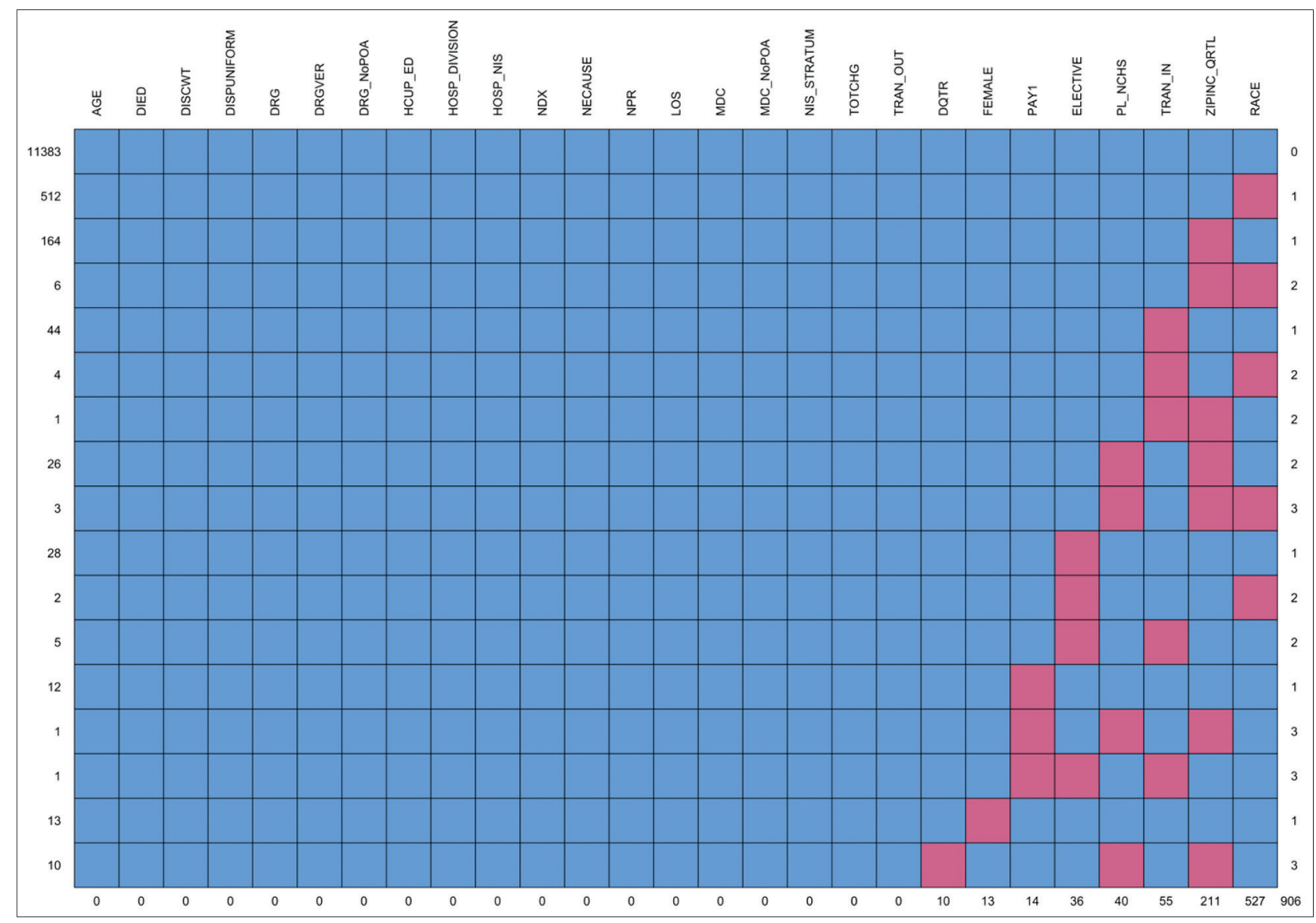

Supplementary Figure 1 Missing data pattern, blue represents no missing data and pink represents missing data

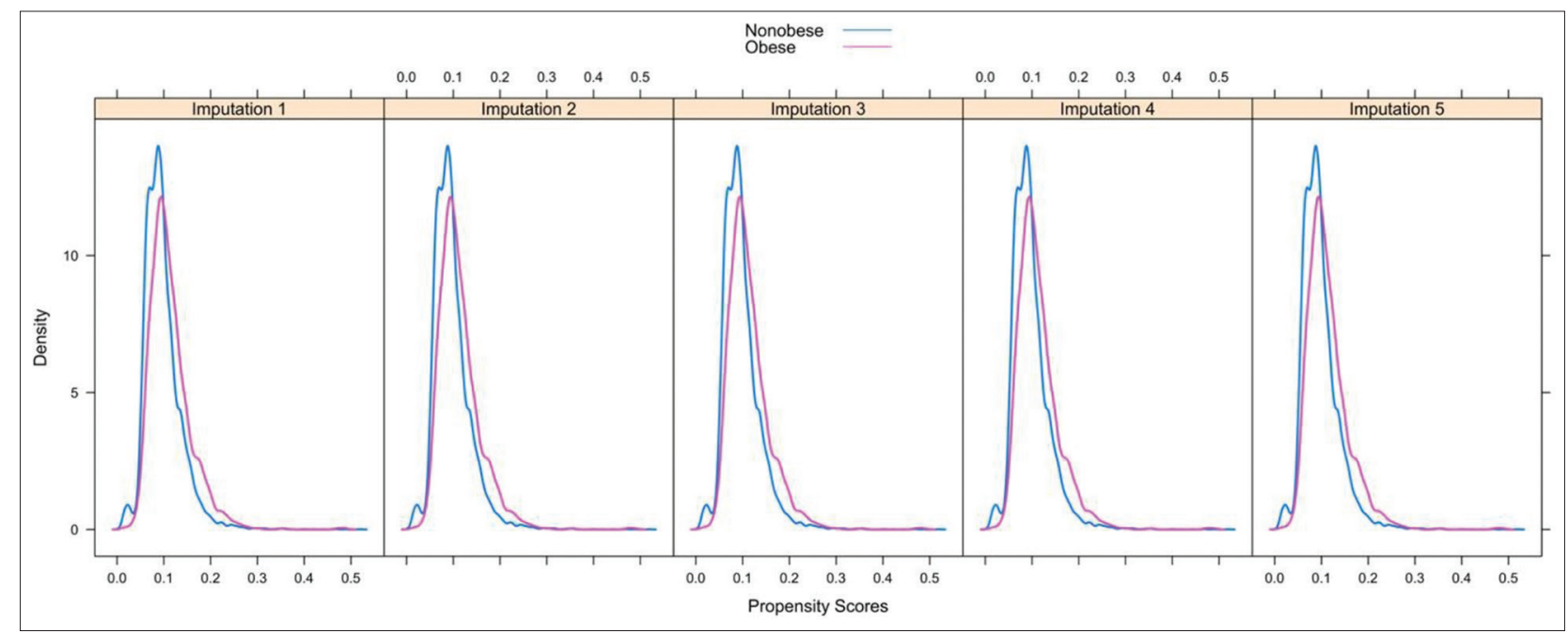

Supplementary Figure 2 Kernel density plots to evaluate common support in the propensity scores estimated by regression model in all imputed datasets. Imputation 1 to Imputation 5 represent all imputed datasets estimated by multiple imputations. The blue curves represent nonobese subjects and pink curves represent obese subjects in the dataset 


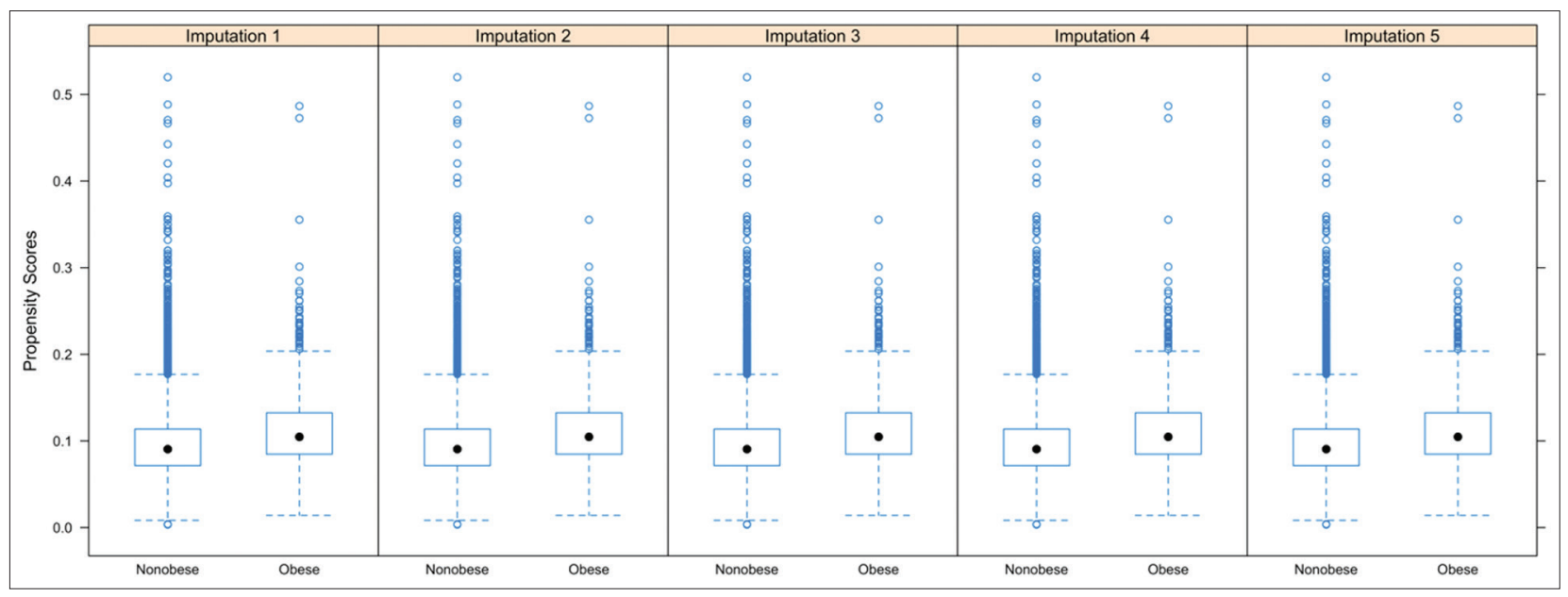

Supplementary Figure 3 Box-and-whiskers plots to evaluate common support in the propensity scores estimated by regression model in all imputed datasets. Imputation 1 to Imputation 5 represent all imputed datasets estimated by multiple imputations

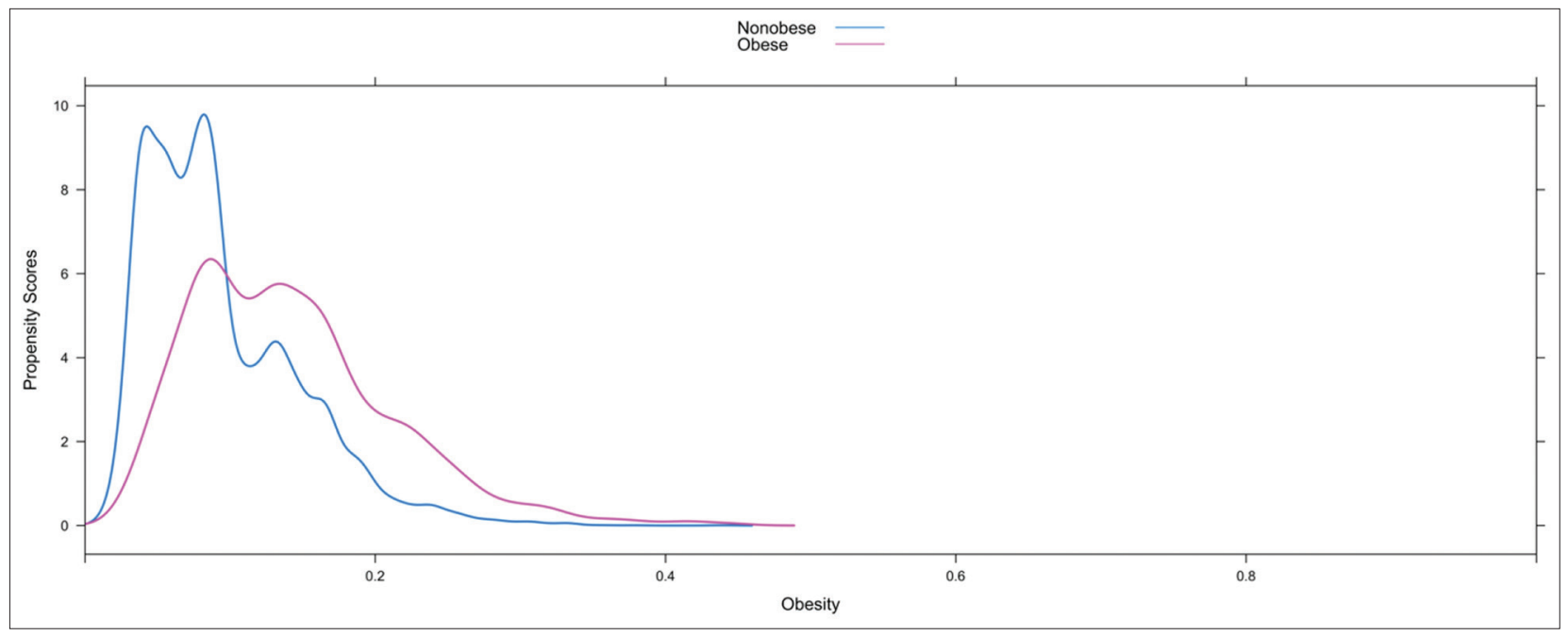

Supplementary Figure 4 Kernel density plot to evaluate common support in the propensity scores estimated by generalized boosting modeling 


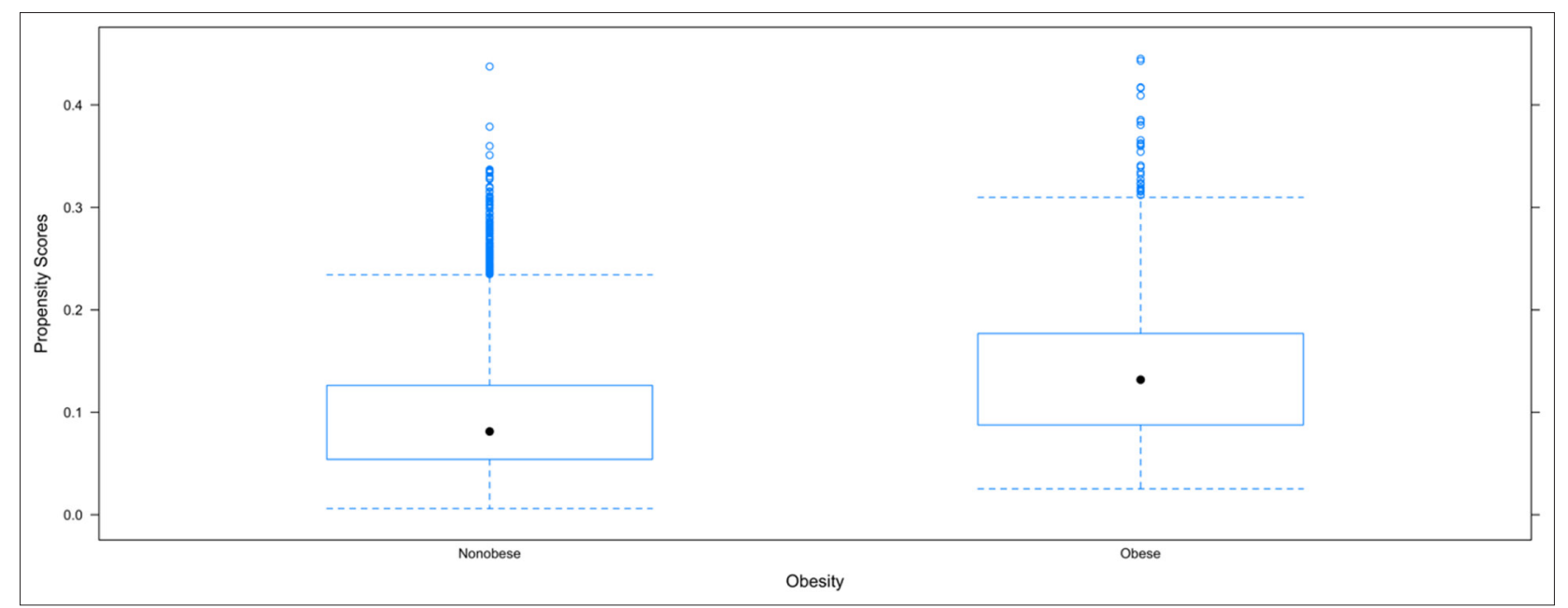

Supplementary Figure 5 Box-and-whiskers plots to evaluate common support in the propensity scores estimated by generalized boosting modeling

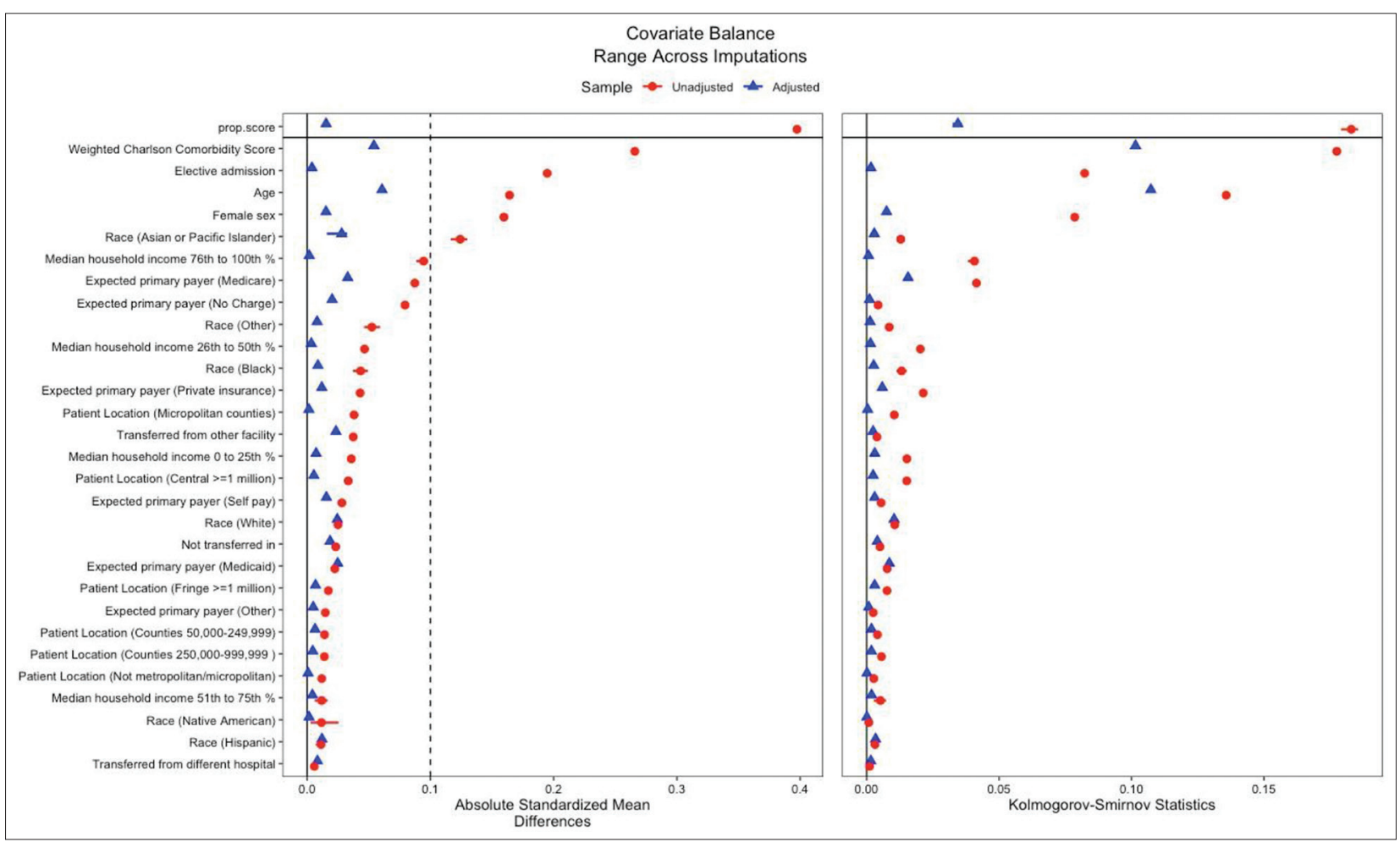

Supplementary Figure 6 Love plot summarizing covariate balance before and after applying inverse probability of treatment weighting (IPTW). - Represent covariates before applying IPTW and $\mathbf{\Delta}$ represent covariates after applying IPTW 


\begin{tabular}{|c|c|c|c|c|c|c|c|}
\hline & MD & $95 \% \mathrm{Cl}$ & P-value & & & & \\
\hline Exclude $C D$ and other types of colitis & 0.55 & $0.18-0.93$ & 0.003 & & & & \\
\hline Exclude overweight & 0.68 & $0.30-1.06$ & 0.001 & & & $=$ & \\
\hline Patients who are 65 years old & 0.59 & $0.17-1.01$ & 0.006 & & & & \\
\hline \multirow[t]{3}{*}{ All patients } & 0.57 & $0.22-0.93$ & 0.002 & & & & \\
\hline & & & & 0 & 0.25 & 0.75 & 1.25 \\
\hline & & & & \multicolumn{4}{|c|}{ Days } \\
\hline
\end{tabular}

Supplementary Figure 7 Subgroup analyses of length of hospital stay $C I$, confidence interval; $M D$, mean difference; $C D$, Crohn's disease

\begin{tabular}{|c|c|c|c|c|}
\hline & MD & $95 \% \mathrm{Cl}$ & P-value & \\
\hline Exclude $C D$ and other types of colitis & 6198 & $2087-10309$ & 0.003 & \\
\hline Exclude overweight & 7164 & $3152-11175$ & 0.0005 & - \\
\hline Patients who are 65 years old & 6710 & $2108-11313$ & 0.004 & \\
\hline \multirow[t]{3}{*}{ All patients } & 6342 & $2500-10184$ & 0.001 & \\
\hline & & & & 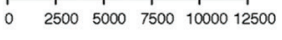 \\
\hline & & & & American Dollars \\
\hline
\end{tabular}

Supplementary Figure 8 Subgroup analyses of total charges $C I$, confidence interval; $M D$, mean difference; $C D$, Crohn's disease 